\title{
How Long Does a Santa Barbara Divorce Take?
}

\author{
SAMUEL J. FRAME
}

BRIAN H. BURKE

\begin{abstract}
There has been considerable research about the amount of time the psychological grief process requires when couples divorce. However, there has been little research on the time the actual process of divorce requires. To address this, we obtained free and publicly available information on divorce cases from Santa Barbara County. We are able to offer some insight about the relationship among the length of divorce, marriage length, and having minor children. Our results are consistent with those found in other literature that focuses on the length of the grief process, and our results are consistent with our experiences in family law practice and mediation.
\end{abstract}

At some point during a divorce, clients might ask, "How long is this divorce going to take?" The question might be asked of a counselor, a family law lawyer, or mediator. Few have any answers supported with evidence. Instead, the response to this important question will be answered on the basis of impressionistic and anecdotal clinical experience. An unqualified answer would be misleading at best. Using data and statistical analysis, we offer some valuable insights to the common and motivational question. Our intuitive and understandable results might be valuable insights for individuals in family law practice or mediation. 
The literature on the length of the divorce process is both limited and equivocal. Established researchers have made comments on the subject, but we find no published study that gathers and analyzes the necessary data. Comments are made in passing and without reference to empirical data. However, there is extensive literature addressing the duration of the psychological grief process that occurs during a divorce (Crosby, Lybarger, \& Mason, 1983, 1986). Folberg and Milne (1988) suggested this process could take 2

to 4 years after the legal divorce is complete. The duration of the entire transitional period, including legal proceedings, settlements, and lifestyle adjustment is also well studied (Vaughn, 1980). Kelly and Wallerstein (1980), as well as Johnston and Cambell (1999), suggested the duration of this transitional period to be 18 to 24 months and 2 to 3 years, respectively. Others simply suggest there is "no timetable" (Ahrons, 1994).

Burke (2009) was one of the first to gather the required data and evaluate a hypothesized length of divorce, which is based on lengthy and experienced mediation and legal practice. It is hypothesized that the length of divorce is between 18 and 36 months. This suggests this question: How do we measure the length of divorce?

In this article, we do not develop advanced statistical methodologies ideally suited for our current data idiosyncrasies. We use reliable statistical methodologies that are accessible to individuals without advanced statistics training. Most important, we clarify and define the length of divorce so it is objectively measurable in legal proceedings in most jurisdictions. Using our definition and the data we have gathered, we have found that the average length of divorce is not all that different from the hypothesized range of 18 to 36 months. We find that divorcing couples with minor children have a longer length of divorce, accounting for marriage length. Also, we find that only "short" marriages have increased divorce lengths, accounting for minor children.

We define a method to measure the length of divorce. We discuss the methodology used to collect the data, the specific population the data represent, and case organization. We then describe the statistical modeling approach used to control for marriage length and having minor children at the time of separation. Next, we discuss a methodology to recommend a sample size for future data collections and analysis. We conclude with suggestions for future research.

\section{THE SANTA BARBARA CENSUS}

A population is a well-defined, complete collection of subjects that have attributes that are of interest. A census occurs when each member of the population is surveyed (Devore, 2008). In 2003, we obtained a census of 
all civil cases filed in the Anacapa Division of South County Santa Barbara (Santa Barbara, California) from January 2 to July 11, 1997.

We identified family law cases initiated by a petition for dissolution, nullity, or legal separation. For the first 6 months of 1997, the census resulted in 358 petitions. Each of the petitions was obtained from the court clerk. We recorded the date of marriage, date of separation, and an indicator the couple had minor children (Burke, 2009). Due to the large variation in the way divorce cases are litigated and subsequently resolved, it can be difficult to define and measure the length of a divorce.

Of the 358 cases, 95 were removed from this analysis for one of three reasons. First, dismissals (13 cases) and joint petitions ( 25 cases) were removed from the data and subsequent analysis. Dismissals are usually the result of reconciliation, in which no divorce actually occurs. In the case of joint petitions, cases are concluded before the initial petition is filed because of legal marriage circumstances and prepetition contracts. The remaining cases (57) were removed because no judgment had been reached by the date of census in 2003, 6 years after the initial petition. For the cases resolved by 2003, we recorded the date of judgment found on the court file for each case.

Longitudinal studies are those that monitor subjects over time. At the end of the study, it is possible that some variables might have not been observed because they occurred after the study ends. For our data, 57 cases did not have an observable judgment during the 6 years after filing the initial petition. Such data are referred to as censored data, specifically right censored in this scenario (Little \& Rubin, 1987). For the 57 cases with no judgment, the actual time to judgment is at least 6 years ( 72 months). There are diverse and extensive statistical methods for analyzing censored data (Little \& Rubin), and future studies and analysis will include the censored data. For our work here, we remove these cases.

We define the length of a divorce to be time to judgment (T2J): the amount of time between the date of separation and the date of entry of judgment on substantive issues (measured in months). Recall that not all divorce cases were resolved by way of judgment or even had a judgment in 2003. As such, this changes the population of interest and the resulting data. Our definition of the length of divorce, T2J, is measurable and reproducible in different jurisdictions (Carrillo, Vazquez, \& Evans, 2010).

Our census consists of 263 cases. For the remainder of this article, we refer to these data as sample data and not a census. Recall that the original census of 358 cases only represented the cases in the first 6 months of 1997. Obviously, divorces occurred in the second half of 1997, and in the many years before and after 1997. Moreover, we removed 95 cases for the aforementioned reasons. The 263 cases represent a census of a very specific population: couples from Santa Barbara who filed a divorce petition during the 6-month period, required judgment, and had a judgment within 6 years of filing. We would like our results to be useful and applicable to other 
individuals from other populations. These populations include cases in different years (particularly current or future cases), and in different locations or jurisdictions. Removing cases due to dismissal or joint petition should be done, as they do not constitute the type of divorce we consider. However, removing the censored data presents a large problem of bias.

For the 57 censored cases, the T2J is clearly longer than 6 years ( 72 months). The effect of not including these cases and failing to account for the censored data will likely bias our statistical analysis. We report estimates of the average T2J, and these estimates will be lower than they would be if the censored data are utilized. We realize this is unattractive and problematic, and the reader should be aware of this caveat. We discuss this more later. The data we use, our analysis, and our conclusions could still be useful for individuals practicing family law and mediation. These results are not specific to other populations, but can still serve as an indication of the expected length of divorce.

For each case record, we have the T2J, measured in months, an indicator that the case involved a minor child at the time the petition was filed (child, either yes or no), and the duration of the marriage (duration, measured in years). Like T2J, marriage duration is determined as the amount of time between the date of marriage and filing date of separation. To simplify the presentation of our results, we create a marriage duration categorical variable. Very short marriages are those that lasted less than 1 year, short marriages are between 1 and 5 years, medium marriages are between 5 and 10 years, and long marriages are more than 10 years. This marriage duration configuration will slightly increase the complexity of the statistical models we consider. However, our categorical marriage duration will simplify the interpretation and presentation of results. Our analysis uses multiple regression models that have the capability of incorporating categorical variables (e.g., duration and child) to model and estimate the average time to judgment.

\section{REGRESSION ANALYSIS}

The statistical analysis we conduct has three purposes. First, it allows us to investigate the potential relationship among marriage duration, having a minor child, and T2J. Experience and common knowledge might suggest that marriages of different durations or with minor children could result in differing lengths of divorce. Next, we use our statistical analysis to estimate the average T2J accounting for marriage duration and having minor children. Finally, we use the results of our statistical analysis to suggest reasonable sample sizes for future research.

A multiple regression model is a natural, common, and reliable statistical model for achieving all of these goals (Neter, Kutner, Nachtsheim, 
\& Wasserman, 1996). Multiple regression is a method for finding the best statistical fit between one variable (e.g., T2J) and other variables (e.g., marriage duration and minor children). We use multiple regression as the basis for this analysis because it is capable of incorporating multiple predictor variables (marriage duration and minor children), identifying possible interactions, and there exist reliable methods to compare candidate models. In this section, we do not give a lengthy exposition of multiple regression models, estimation methods, variable selection, or model comparison methods (see Neter et al., 1996). All computations were done using the R Statistical Computing Environment (Maindonald and Braun, 2007; Verzani. 2000).

For this analysis, we consider various candidate regression models with different configurations and complexities. The first model we consider uses a minor children indicator and the categorical marriage duration to predict T2J (with the long marriage duration as the base or reference group.

$$
T 2 J=\beta_{0}+\beta_{1} \text { Child }+\beta_{2} \text { Medium }+\beta_{3} \text { Short }+\beta_{4} \text { VeryShort }+\varepsilon
$$

In Equation 1, $\beta_{0}$ is the average T2J for long marriages without minor children (i.e., the intercept), $\beta_{1}$ is the average change in T2J for minor children (accounting for marriage length), $\beta_{2}$ through $\beta_{4}$ are the average changes in T2J for marriages of medium, short, and very short lengths, respectively (accounting for minor children), and $\varepsilon$ is random error.

With a sample of 263 , we can easily estimate the parameters of this regression model (e.g., the $\beta$ coefficients). A summary of the estimated regression model is given in Table 1 . Accounting for marriage duration, the estimated average T2J increases by over 14 months for couples with minor children, and this increase is statistically significant. Interestingly, the estimated regression model suggests that the estimated average T2J is significantly different (in this case larger) for short marriages only, accounting for minor children.

To better establish the importance of marriage duration, we consider an alternative regression model that only uses the minor child indicator and a short marriage indicator. For marriage duration, we hypothesize two groups. The first group combines very short, medium, and long marriages

TABLE 1 Equation 1 Estimated Regression Model

\begin{tabular}{lrrrr}
\hline Coefficient & Estimate & $S E$ & $t$ & $p$ value \\
\hline Intercept & 21.33 & 3.98 & 5.36 & .00 \\
Child yes & 14.18 & 3.73 & 3.80 & .00 \\
Duration medium & -1.38 & 4.64 & -0.30 & .77 \\
Duration short & 7.95 & 4.58 & 1.74 & .08 \\
Duration very short & -3.17 & 7.28 & -0.44 & .66 \\
\hline
\end{tabular}


into a single group. The second group only consists of short marriages. This model is nested inside of Equation 1, and we use a standard analysis of variance partial $F$ test to compare these two models (Neter et al., 1996). The $p$ value for the model comparison is .8969 , which indicates there is no evidence the larger model is better. From this, we are able to conclude the estimated average T2J is significantly longer for short marriages (accounting for minor children).

We do consider two other alternative candidate regression models. First, we consider a further reduced regression model that only uses minor children to predict T2J. This is the regression model analogue of a twosample $t$-test (Neter et al., 1996). The estimated regression model suggests a very similar relationship between minor children and the average T2J. Comparison of this smaller model to the estimate regression model in Equation 1 gives a $p$ value of .02111, which suggests that the short marriage duration indicator is needed in the model and helps to explain the variation in T2J values.

In an effort to explore a more complex regression model, we consider a model that includes all of the marriage durations, minor children, and interactions between these variables to predict T2J. This would allow for the possibility that there is a different relationship between marriage duration and the average T2J with and without minor children. We find no utility with the additional marriage durations and interaction terms (the $p$ value for model comparison is .864).

Although we can conclude only short marriage durations and having minor children are important for understanding T2J, using marriage duration does provide more specific estimates of the average T2J. For example, clients will want to know the estimated average T2J for their particular length of marriage (regardless of the analysis we present here). Table 2 gives the estimated average T2J and 95\% confidence intervals for each group defined by marriage duration and having minor children. These values demonstrate the implications of the regression analysis and model selection process. It is clear that marriages with minor children, short durations, or both have a longer length of divorce.

The estimated average T2J is graphically presented in Figure 1. Visually, it is clear that divorces are longer for couples with minor children. The visual representation also demonstrates how short marriages have a longer length of divorce (as indicated by the respective spikes for short marriages). We have found Figure 1 to be extremely useful for counseling and mediation purposes when clients ask "How long will this divorce take?" With this graph, individuals can classify their marriage duration and minor children status, and visually obtain a rough estimate of how long the divorce will take.

The results we present here are for our specific set of 263 cases, and are directly applicable to the specific Santa Barbara population. For different time periods and jurisdictions, these results could be useful with the caveats 
TABLE 2 Estimated Average Times to Judgment and 95\% Confidence Intervals (in Months)

\begin{tabular}{lccc}
\hline Duration & Minor Children & Estimate & Confidence Interval \\
\hline Very short & No & 16.73 & {$[2.13,31.34]$} \\
Short & No & 27.82 & {$[20.33,35.32]$} \\
Medium & No & 23.13 & {$[14.07,32.19]$} \\
Long & No & 20.61 & {$[9.92,31.30]$} \\
Very short & Yes & 36.60 & {$[11.30,61.90]$} \\
Short & Yes & 45.62 & {$[35.63,57.41]$} \\
Medium & Yes & 30.36 & {$[20.52,40.21]$} \\
Long & Yes & 35.85 & {$[28.48,43.21]$} \\
\hline
\end{tabular}

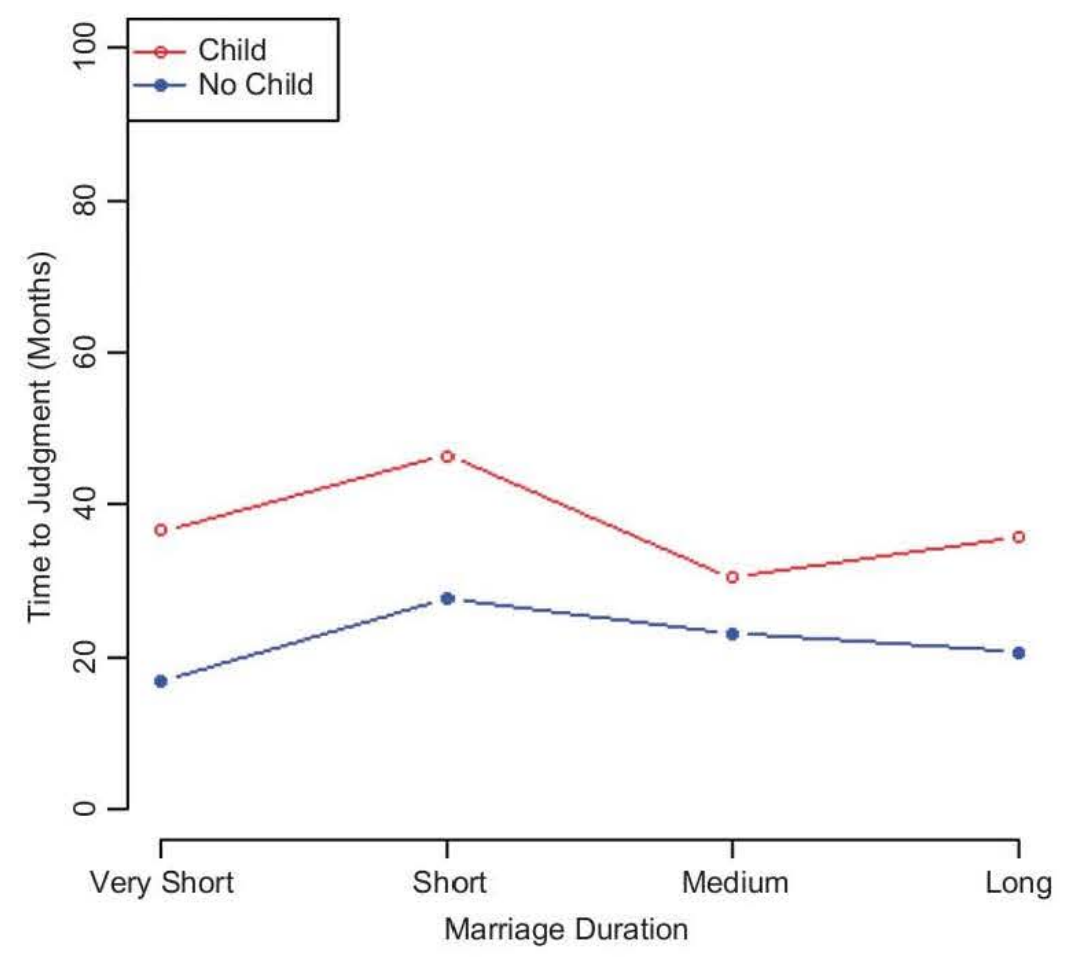

FIGURE 1 Estimated average time to judgment by duration and child (color figure available online).

we have discussed. Suppose a researcher wants to conduct a similar study and is going to gather new data. How large of a random sample size should he or she acquire for the results to be reliable? In the next section, we address the question of sample size determination for future studies.

\section{SAMPLE SIZE DETERMINATION}

The data we have gathered and analyzed offer new insights for individuals practicing family law and mediation. However, this work only constitutes 
preliminary research and should be extended further. Researchers might be interested in obtaining data from other jurisdictions. In our experience, gathering these data can be time consuming and might be costly. Future data collections should use conservative sample sizes that are large enough to reliably estimate model parameters.

Sample size determination is closely linked to estimating population parameters. In fact, it answers the question, "What type of sample size is needed to estimate the population average T2J, to within some precision, with some probability?" Our multiple regression model yields an estimate of the conditional variance of the T2J values. The estimate of variability is important for any sample size determination. Populations with larger variation will require more samples to estimate parameters with the same level of accuracy when compared to populations with less variation.

Sample size determination is widely studied in the statistics community. Park and Dudycha (1974) were among the first to consider the problem in the regression context, and much work has been done since. Here, we present a straightforward approach to suggest a sample size. This approach can be used to suggest sample sizes for different years and jurisdictions other than Santa Barbara, under certain assumptions. If researchers have timely estimates of the conditional variance specific to jurisdictions other than Santa Barbara, those should be used in place of the estimate we use here. The method for suggesting a sample size for other years and jurisdictions will not be different from what we present here.

To estimate the population average T2J within some precision, $\delta$, with some probability, $1-\alpha$, Park and Dudycha (1974) suggest a sample size given by

$$
N_{0}=\frac{s^{2} T_{N-3, \frac{\alpha^{2}}{2}}}{\delta^{2}}
$$

where $s^{2}$ is the estimated conditional variance and $T_{N-3, \frac{\alpha}{2}}$ is the $1-\frac{\alpha}{2}$ percentile of a $T$ distribution with $N-3$ degrees of freedom. However, this assumes that the population is infinitely large. For finite populations of size $N$, we correct this by

$$
N^{*}=\frac{N_{0} N}{N_{0}+N-1}
$$

to give a suggested sample size.

First, we consider how the suggested sample size increases with precision. The precision, $\delta$, is how close estimates are to the true population value (in absolute value). Increasing precision means that $\delta$ becomes smaller, and requires more samples. Table 3 gives sample size suggestions for 
TABLE 3 Suggested Sample Size

\begin{tabular}{lc}
\hline Precision (in Months) & Suggested Sample Size \\
\hline 0.5 & 241 \\
1 & 190 \\
3 & 60 \\
6 & 18 \\
\hline
\end{tabular}

Santa Barbara with a finite population of divorce cases (263) and with probability .95 .

Estimating the average T2J more precisely requires more sample data. We find a sample size of 60 to be sufficient for reliable estimation. This is consistent with sample data replication studies already done (Burke, 2009). In situations where data have to be manually inspected and organized, it would be much easier to manage 60 cases rather than hundreds or possibly thousands.

The preceding suggestion is specific to the Santa Barbara population. Researchers in different jurisdictions can utilize these suggestions, assuming different jurisdictions have population characteristics that are not different from Santa Barbara. Specifically, a researcher has to assume the relationship among marriage duration, minor children, and T2J is similar. Also, it must be assumed that the conditional variance is similar.

Burke (2009) obtained data from Santa Barbara for cases filed in 2003, and found the characteristics to be comparable to the cases filed in 1997. This suggests the results from 1997 are reasonably applicable to Santa Barbara in other years. In the spring of 2010, students at California Polytechnic State University gathered data from San Luis Obispo consisting of 75 cases filed in 2003 (Carrillo et al., 2010). Their initial findings indicate that the Santa Barbara and San Luis Obispo populations are remarkably similar. This might be explained by the geographic and demographic similarities between the two counties.

\section{CONCLUSIONS AND FUTURE RESEARCH}

The primary objective of this work is not to develop or employ advanced statistical methods, or to use complicated and geographically diverse census or sampling techniques over time. Here, we obtain free, publicly available data and use standard statistical methods to determine how long a Santa Barbara divorce takes. The regression analysis we use and the method for suggesting a future sample size are both based on the assumption that the T2J values follow a normal distribution (Devore, 2008; Neter et al., 1996).

Although we did not present the results here, the T2J values do not follow a normal distribution. Strictly speaking, we should use alternative 
methodologies that reflect this. This adds an additional caveat to our conclusions, such as generalized linear models, which can easily remedy the lack of normality. Also, recall that many of the cases are right censored. In future studies, this should be accounted for by using survival analysis methods.

All the same, these results give family law litigators and mediators some indication of the length of divorce. It was originally hypothesized that divorces could take between 18 and 36 months. Our results are consistent with this hypothesis. We find that marriages with minor children, short durations, or both have a significantly increased length of divorce.

\section{REFERENCES}

Ahrons, C. (1994). The good divorce. New York, NY: HarperColllins.

Burke, B. H. (2009). Santa Barbara divorce: A six-year longitudinal study. Santa Barbara, CA: Brian H. Burke \& Cholmondeley. Retrieved from http://web. me.com/santabarbaraproject/Santa_Barbara_Divorce_Project/CONTENTS_.html

Carrillo, A., Vazquez, J., \& Evans, M. (2010). The ugly truth: Divorce in SLO (STAT 417: Survival Analysis, Project Report). San Luis, Obispo, CA: California Polytechnic State University.

Crosby, J. F., Lybarger, S. K., \& Mason, R. L. (1983). The grief resolution process in divorce. Journal of Divorce, 7, 3-18.

Crosby, J. F., Lybarger, S. K., \& Mason, R. L. (1986). The grief resolution process in divorce: Phase II. Journal of Divorce, 10, 17-40.

Devore, L. J. (2008). Probability and statistics for engineering and the sciences (7th ed.). Belmont, CA: Thompson Higher Education.

Folberg, J., \& Milne, A. (1988). Divorce mediation: Theory and practice. New York, NY: Guilford.

Johnston, J. R., \& Campbell, L. E. G. (1999). Impasses of divorce. New York: NY: Simon \& Schuster.

Kelly, J. B., \& Wallerstein, J. S. (1980). Surviving the breakup. Washington, DC: Library of Congress.

Little, R. J. A., \& Rubin, D. B. (1987). Statistical analysis with missing data. New York, NY: Wiley.

Maindonald, J., \& Braun, W. J. (2007). Data analysis and graphics using R: An example-based approach (2nd ed.). Cambridge, UK: Cambridge University Press.

Neter, J., Kutner, M. H., Nachtsheim, C. J., \& Wasserman, W. (1996). Applied linear statistical models (4th ed.). Boston, MA: WCB/McGraw-Hill.

Park, C. N., \& Dudycha, A. L. (1974). A cross-validation approach for sample size determination for regression models. Journal of the American Statistical Association, 69, 214-218.

Vaughn, D. (1980). Uncoupling. New York, NY: Random House.

Verzani, J. (2000). Using R for introductory statistics. Boca Raton, FL: Chapman \& Hall/CRC Press. 